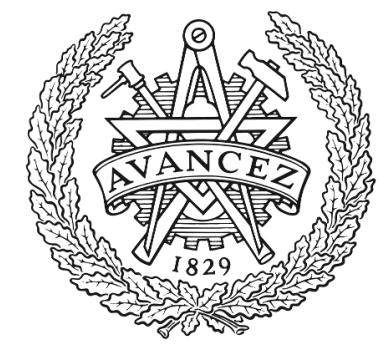

\title{
CHALMERS
}

UNIVERSITY OF TECHNOLOGY

\section{Experimental and Numerical Investigation of the Cavitating Flows over a Modified NACA0009 Foil}

Downloaded from: https://research.chalmers.se, 2023-04-26 13:13 UTC

Citation for the original published paper (version of record):

Arabnejad Khanouki, M., Amini, A., Bensow, R. et al (2018). Experimental and Numerical Investigation of the Cavitating Flows over a Modified NACA0009 Foil. Proceedings of the 10th International Symposium on Cavitation (CAV2018). http://dx.doi.org/10.1115/1.861851_ch71

N.B. When citing this work, cite the original published paper. 


\title{
Experimental and Numerical Investigation of the Cavitating Flows over a Modified NACA0009 Foil
}

\author{
${ }^{1}$ Mohammad Hossein Arabnejad*; ${ }^{2}$ Ali Amini; ${ }^{1}$ Rickard Bensow; ${ }^{2}$ Mohamed Farhat; \\ ${ }^{1}$ Chalmers University of Technology, Gothenburg, Sweden; ${ }^{2}$ Ecole Polytechnique Federale de Lausanne, Lausanne, Switzerland
}

\begin{abstract}
In this study, the cavitating flow over a modified NACA0009 foil has been investigated using experimental and numerical methods. In the experimental investigation, high-speed visualization is used to study the behavior of the cavitating flow at $\sigma=1.2, \alpha=5^{\circ}, U_{\infty}=20 \mathrm{~m} / \mathrm{s}$. In order to identify the location of erosive collapse, a preliminary soft paint test is performed. In the numerical part of this study, the flow condition subjected to the soft paint study is simulated with Large Eddy Simulation using a mixture assumption coupled with the Schnerr-Sauer mass transfer model. In order to validate the simulation, the numerical results are compared with experimental high-speed visualization of the flow at the same condition. These comparisons show that the numerical simulation is capable of reproducing the main features of the cavitating flow. The validated numerical results and the high-speed visualization are then used to explain the hydrodynamic mechanism of erosive events that have been identified in the experimental investigation.
\end{abstract}

Keywords:

\section{Introduction}

Cavitation erosion is the material loss due to the collapse of vapour structures close to the surface. In hydraulic machinery, cavitation erosion is one of the limiting factors in the design as it is usually associated with performance degradation and increase in maintenance costs. In the path towards the prediction of cavitation erosion, understanding the hydrodynamic mechanism of cavitation erosion is essential. Several studies have been devoted to identifying these hydrodynamic mechanisms using experimental observation. Bark and Bensow [1] outlined different hydrodynamic mechanisms that influence the erosiveness of a collapsing cavity. A set of analysis models for these hydrodynamic mechanisms were established using experimental observation complemented by highfidelity numerical simulation. Dular and Petkovsek [2] studied the contribution of different cavitating structures in the cavitation erosion using synchronous observation of cavitation structure and erosion damage. They identified five different mechanisms of cavitation erosion. These mechanisms are spherical collapse of the cloud cavity, the collapse of horse-shoe vortex, the collapse of twister vortex, collapse of microbubbles at the closure of the sheet cavity, and the collapse of microcavities at the break-up region of the cloud cavity. They concluded that the collapse of the cloud cavitation structures is responsible for 80 percent of the erosion damage. Van Rijsbergen et al. [3] used high-speed visualization, paint test, and acoustic measurement to investigate the behavior of the cavitating flow over a NACA0015 foil with the aim to identify the role of different cavitating structures in the cavitation erosion. Similar to Dular and Petkovsek [2], they found that the collapse of cavitating cloudy structures is associated with high risk of cavitation erosion. More recently, Cao et al. [4] investigated the relationship between cavitation structure and erosion damage on a twisted foil using high-speed visualization and paint test. They observed a severe paint removal close to the closure line of sheet cavity where the unsteadiness of the rear part of the sheet cavity leads to the detachment and the collapse of cavity structures.

With the progress in numerical modeling of cavitating flows, high-end numerical simulations have become a useful tool to study the main features of cavitating flows. Numerical simulations provide complete access to the flow field that can be used to analyze the development of cavitating structures. These numerical analyses combined with the experimental observations promote the understanding hydrodynamic mechanisms of cavitation erosion. In this paper, numerical simulations, high-speed visualization, and paint test are employed together to study the cavitating flow over a NACA0009 foil. Based on the simulation results and the experimental images, cavitating structures responsible for the paint removal in the paint test are identified and their hydrodynamic mechanism is discussed briefly.

*Corresponding Author, Mohammad Hossein Arabnejad: mohammad.h.arabnejad@chalmers.se 

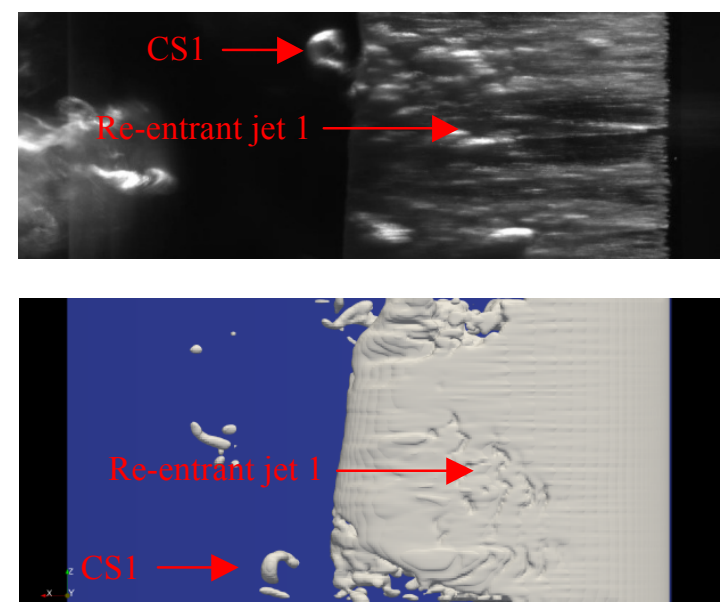

(a) $t=t_{1}$
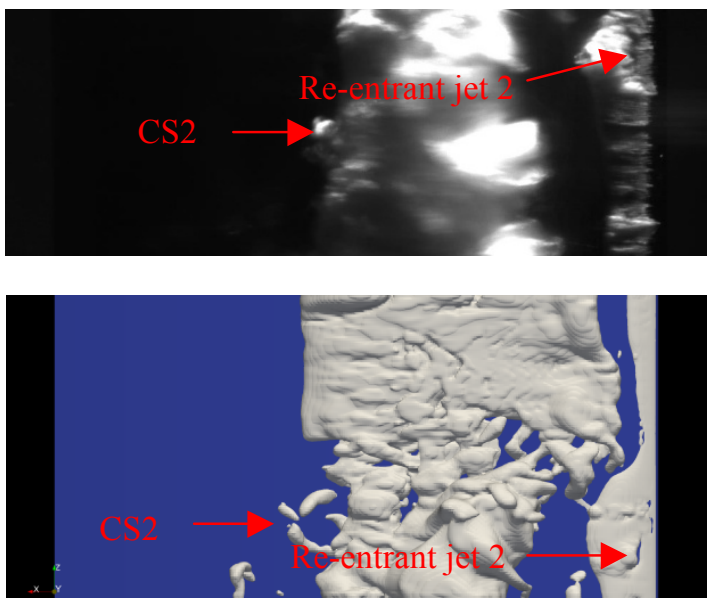

(c) $t=t_{1}+2 / 5 T$
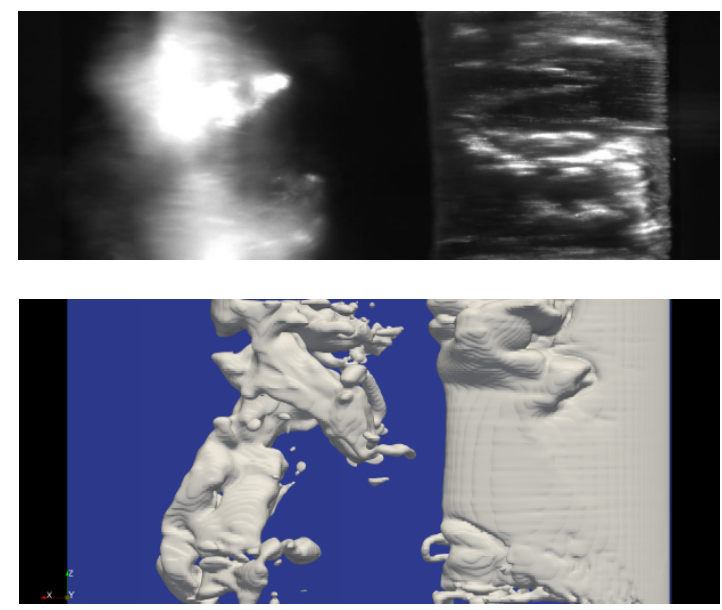

(e) $t=t_{1}+4 / 5 T$
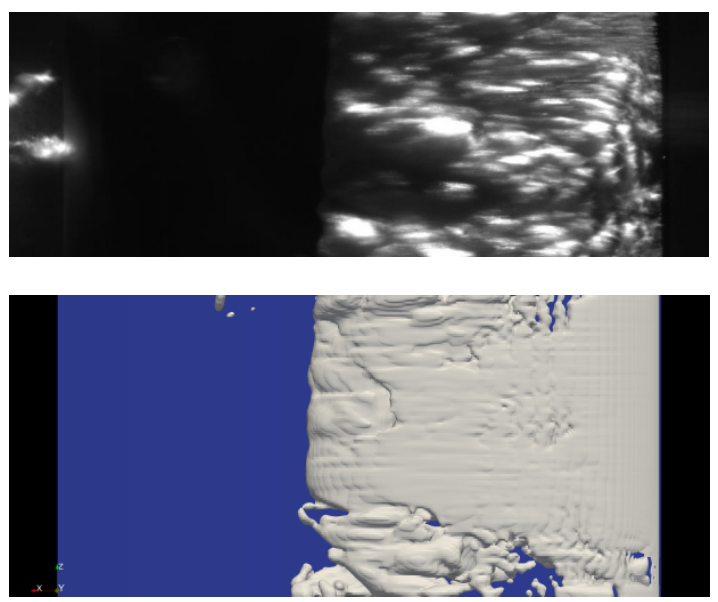

(b) $t=t_{1}+1 / 5 T$
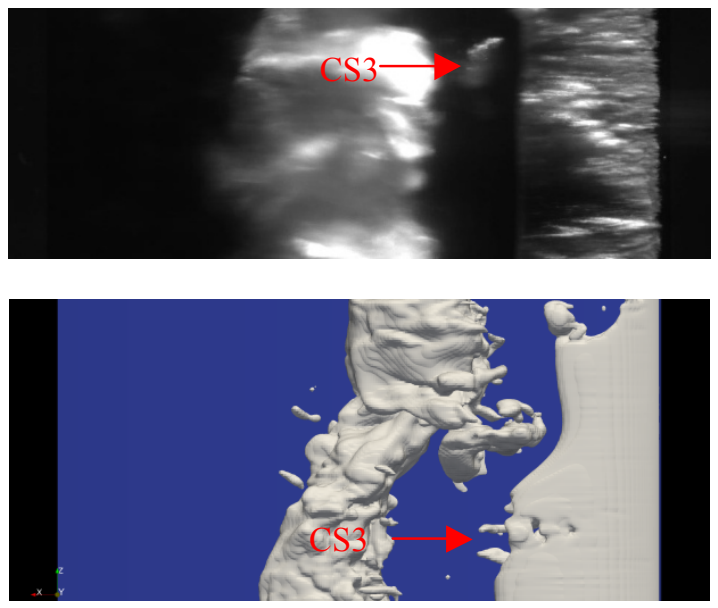

(d) $t=t_{1}+3 / 5 T$
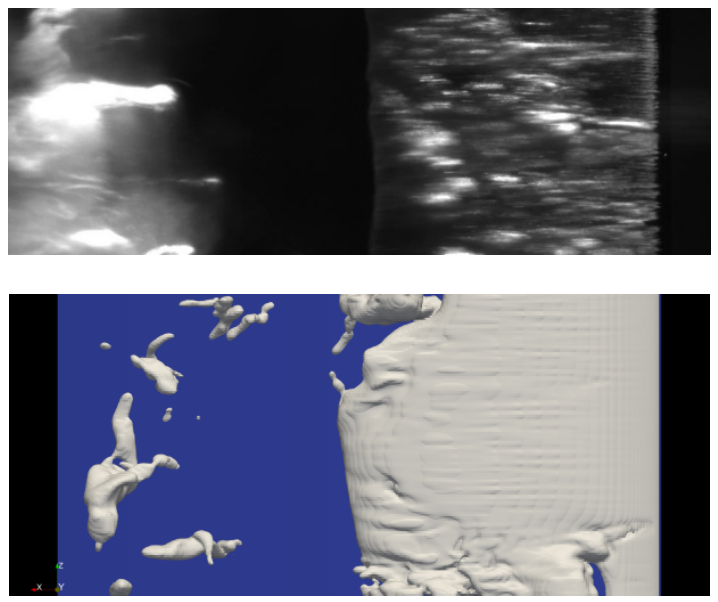

(f) $t=t_{1}+T$

Figure 1: Shedding of the cloud cavitation in one cycle, top) experimental high-speed visualization, bottom) numerical results 


\section{Experimental and Numerical Set-up}

The experimental work presented here has been conducted in the EPFL cavitation tunnel. A modified NACA0009 foil is placed in the cavitation tunnel with $5^{\circ}$ angle of attack. The foil has a chord length of $100 \mathrm{~mm}$ and span length of $150 \mathrm{~mm}$. The cavitation number is 1.2 , and the inlet velocity is $20 \mathrm{~m} / \mathrm{s}$. High-speed visualization and paint test are used to record the cavitation dynamics and identify areas with high risk of cavitation erosion. In the numerical part of this study, the flow has been simulated at the same conditions with Large Eddy Simulation method, using a mixture assumption coupled with the Schnerr-Sauer mass transfer model [6]. The computational grid is composed of 7.4 million hexahedral/prismatic cells. The average value of $y^{+}$around the foil is less than 1 , and the maximum value of $x^{+}$on the upper surface of the foil is around 300. In the span-wise direction, a uniform mesh with $\mathrm{z}^{+}$around 300 is used; thus the cavitation dynamics is well resolved although the surface mesh is not fully sufficient for a wall resolved LES. To decrease the computational cost, the computational domain includes only one-third of the foil span and slip boundary conditions are applied to the side planes.

\section{Results}

Figure 1 shows the shedding of the cloud cavitation during one cycle in both experimental and numerical investigations. In figure 1a, the sheet cavity has reached its maximum length, which is $55 \%$ of the chord length. At this stage, a re-entrant jet has been developed from the closure line of the sheet cavity. The front line of this reentrant jet is marked by red arrows in figure 1a. As the re-entrant jet moves towards the leading edge, it creates disturbances on the interface of the sheet cavity. The disturbances on the surface of the sheet cavity can become larger and lead to the detachment of cavity structures. These structures are marked as CS1 in figure 1a. In figure $1 \mathrm{~b}$, the re-entrant jet has reached the leading edge and caused the pinch-off of the cloud cavity. The cloud cavity has a rolling motion due to the interaction between the re-entrant jet and the bulk flow. As the cloud cavity rolls up, some vapor structures of horse-shoe shape are detached from the trailing part of the cloud cavity. In figure $1 \mathrm{~b}$ and $1 \mathrm{c}$, these structures, marked as CS2, collapse as they travel downstream. In figure 1c, the new sheet cavity has appeared at the leading edge. While the sheet cavity is growing, another re-entrant jet moves upstream and reaches the leading edge. The front line of this re-entrant jet is marked by red arrows in figure 1c. This re-entrant jet does not lead to the pinch-off of the cavity, but it only creates a disturbance on the interface of the sheet cavity. This disturbance leads to the detachment and collapse of vapor structures (marked by CS3 in figure 1d) close to the closure line of the sheet cavity. In figures $1 \mathrm{f}$ and $1 \mathrm{e}$, the sheet cavity grows to its maximum length and the cycle repeats. The comparison between the numerical simulation results and the experimental high-speed visualization shows that the numerical simulation with the current set-up and grid resolution is able to capture the main features of

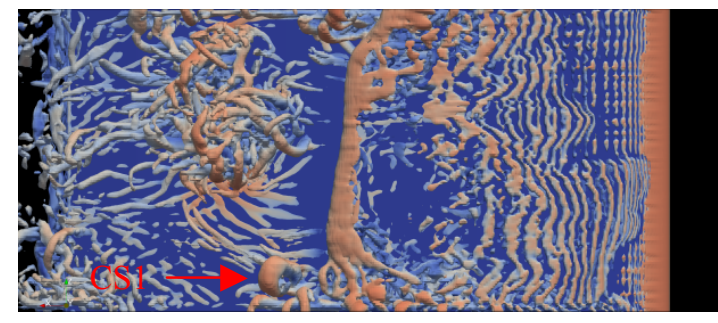

(a) $t=t_{1}$

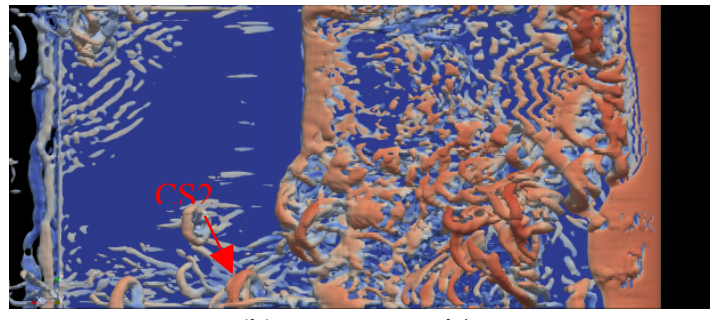

(b) $t=t_{1}+2 / 5 T$

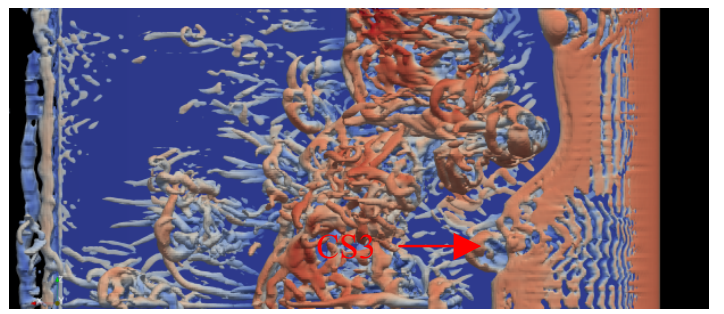

(c) $t=t_{1}+3 / 5 T$

Figure 2: Q criteria in one shedding cycle 
the cavitating flow. In the experiment results, the shed cloud turns into a $U$ shape as it moves downstream while in the numerical results the cloud does not have any particular shape. The reason for this difference might be that the side walls in the simulation are defined as slip boundary condition, therefore the effect of the side walls are not present.

In figure 2, the vortical structures for three time instances are shown by the iso-surface of the $\mathrm{Q}$ criterion. These time instances corresponds to figure 1a, figure 1c, and figure 1d. As it can be seen from this figure, the structure CS1, CS2, and CS3 are horse-shoe vortical structures detached from the closure line of the sheet cavity or the downstream end of the cloud cavity.

In order to locate the areas with high risk of cavitation erosion, a stencil ink is applied to the surface of the foil. Figure 3a shows the status of the stencil ink after 10 minutes and figure $3 \mathrm{~b}$ shows the span-wise average of the paint removal. A region with severe paint removal is observed in the stream-wise location of $0.3<\mathrm{X} / \mathrm{C}<0.7$. This region coincides with the collapse location of cavity structures CS1, CS2, and CS3, identified in figure 1. This suggests that the cavity structures detached from the closure line of the sheet cavity and the downstream end of the cloud cavity can be responsible for paint removal seen in figure 3 .

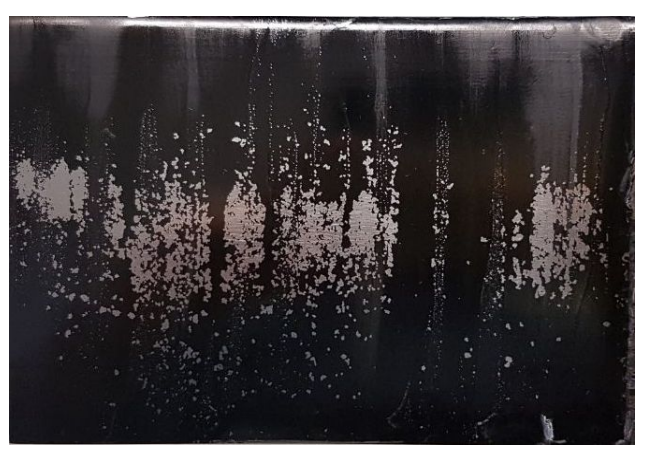

(a)

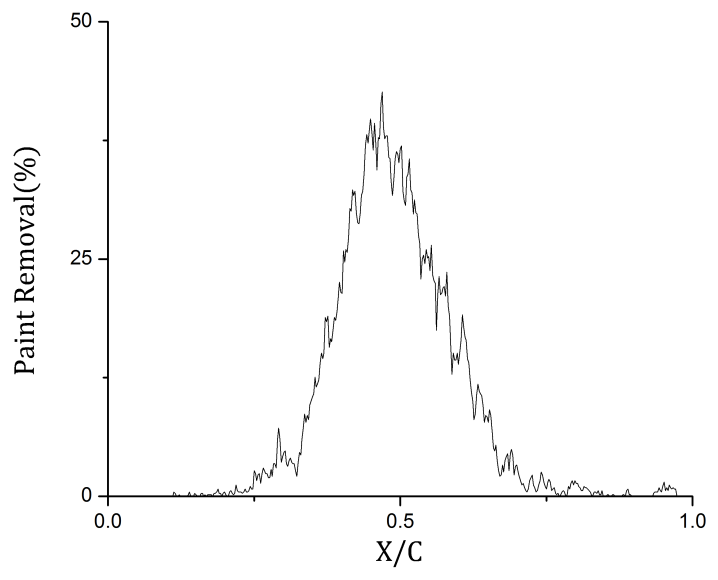

(b)

Figure 2: a) Paint removal after 10 minutes. b) Span-wise average of paint removal as a function of streamwise location

\section{Conclusion}

This paper presents the numerical and experimental investigation of a cavitating flow over a modified NACA0009 foil. The behavior of the flow is studied by experimental high-speed visualization and numerical simulation results. This study shows that smaller horseshoe cavitating structures are detached from the closure line of the sheet cavity and downstream end of the cloud cavity. These structure collapse shortly after their detachment. The paint test results show that most paint removal occurs in the area close to the collapse of the identified horse-shoe structures. This suggests that the collapse of cavitating structures detached from the closure line of the sheet cavity and downstream end of the cloud cavity can be responsible for paint removal.

\section{Acknowledgments}

Financial support of this research is provided by the EU CaFE initiative.

\section{References}

[1]Bark, G., and Bensow, R. E., (2013), Hydrodynamic Mechanisms Controlling Cavitation Erosion,Int. Shipbuild. Prog., 60(1)

[2]Dular, M. and Petkovsek, M., (2013). On the mechanisms of cavitation erosion-Coupling high speed videos to damage patterns. Wear (300). 
[3] van Rijsbergen, M., Foeth, E.J., Fitzsimmons, P., Boorsma, A.,(2012)High speed video observations and acoustic impact measurements on a NACA0015 foil, Proceedings of the 8th International Symposium on Cavitation, Singapore.

[4] Cao, Y.T., Peng, X.X., Yan, K., Xu, L.H., and Shu L.W, (2017) A qualitative study on the relationship between cavitation structure and erosion region around a $3 d$ twisted hydrofoil by painting method. Fifth International Symposium on Marine Propulsors, Espoo, Finland.

[5]Bensow, R. E., and Bark, G., (2010). Implicit LES Predictions of the Cavitating Flow on a Propeller. Journal of Fluids Engineering, Vol. 132.

[6]Asnaghi, A., Feymark, A., and Bensow, R.E., (2017). Improvement of cavitation mass transfer modeling based on local flow properties. International Journal of Multiphase Flow, Vol. 93. 